\title{
Performance Analysis for Audio Streaming in Cloud
}

\author{
${ }^{1}$ Pramod Kumar, ${ }^{2}$ Dinesh Goyal \\ ${ }^{1,2}$ Suresh Gyan Vihar University
}

\begin{abstract}
Audio streaming has been a major application, which has become popular in past few years especially amongst youth both in professional and personal lives. Various organizations to develop and deploy their own cloud, and their meeting most of them are in the same cloud, holding a variety of audio codecs to be used for real-time streaming.

The concept of cloud computing has been proposed from the device to the cloud computing power, storage, and security unloading resource requirements. Internet audio applications such as real-time streaming is expected to be ubiquitous in the cloud users, they often encounter a diverse range of wireless access technology, wireless networks, roaming and in their closed position and support the work of the deployment. FLAC, MP3 and WAV streams are used for real-time streaming. In this paper, we analyze the various quality parameters, FLAC, MP3 and WAV format audio streams in different environmental conditions, they deliver quality performance
\end{abstract}

Key Words: Cloud, Audio, Streaming, FLAC, MP3

\subsection{Cloud Computing-The Concept}

\section{Introduction}

Cloud computing is Internet-based ("cloud") development and use of computer technology ("computing"). It is a style of computing, dynamically scalable and often virtualized resources are provided as a service over the Internet. Users need not have knowledge of, expertise, or "in the cloud", in support of their control over the technology infrastructure.

This concept includes Infrastructure as a Service (IaaS), Platform as a Service (PaaS) and Software as a Service (SaaS), as well as Web 2.0 and other recent technology trends on the Internet have a common theme depends meet computing to meet the needs of users . Examples of SaaS vendors include Salesforce.com and Google applications provide universal service access from a Web browser, while the software and data are stored on the server of the online application.

The term "cloud" as a metaphor for the Internet, based on how the Internet is depicted in computer network diagrams as an abstract complex infrastructure that hides.

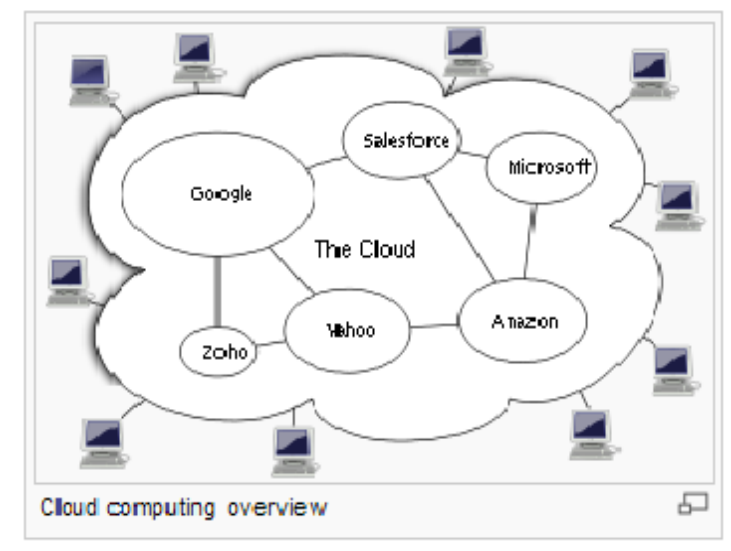

\section{About Audio Formats}

There are many kinds of audio formats which are discussed below:

1. AAC: AAC stands for either MPEG2 Advanced Audio Coding or MPEG4 Advanced Audio Coding. The MPEG2 audio-encoding standard of the format is not backward-compatible with MPEG1 audio. MPEG2 AAC can produce better audio quality than MP3 using less physical space for the files. MPEG4 AAC can produce better quality and smaller files than MPEG2 AAC. AAC is the audio file format used by Apple in their popular iTunes Music Store. Files may appear on your system with the ".M4A" filename extension.

2. AC3: AC3 file, a Dolby Digital audio file, can be found as the standard audio track on Digital Versatile Discs (DVD) and High Definition Television (HDTV). This coder has been designed to take maximum 
advantage of human auditory masking in that it divides the audio spectrum of each channel into narrow frequency bands of different sizes optimized with respect to the frequency selectivity of human hearing. This makes it possible to sharply filter coding noise so that it is forced to stay very close in frequency to the frequency components of the audio signal being coded. By reducing or eliminating coding noise wherever there are no audio signals to mask it, the sound quality of the original signal can be subjectively preserved.

3. AIFF: AIFF stands for Audio Interchange File Format. Apple Computer developed this audio file format to store high-quality audio and musical instrument information.

4. FLAC: FLAC stands for Free Lossless Audio Codec. By encoding audio files with FLAC, the quality is exactly the same as the original audio file's quality is. This is exactly unlike the audio formats such as MP3 and WMA work -- these audio formats are called "lossy" and that means that when the original audio is encoded into the lossy audio format, some of the audio data is lost forever and can't be brought back by any means.

5. M4A: The audio file format used by Apple in their popular iTunes Music Store often appears on your system with the ".M4A" filename extension. M4A can produce better audio quality than MP3 using less physical space for the files. View the bestM4A to MP3 Converter.

6. M4P: M4P format is "protected AAC". It is a format of purchased music that can be listened to only through the iTunes softer or an iPod.

7. MIDI: MIDI stands for Musical Instrument Digital Interface. A MIDI file doesn't contain actual audio data, but rather contains commands that let MIDI-capable synthesizers re-create a specific musical passage. The MIDI protocol has been used for years as a way for electronic musical instruments (like digital keyboards and sequencers) to communicate with each other. Computer sound cards typically feature the ability to interpret MIDI files into music. Since they don't actually contain the music itself, but rather the commands used to re-create music, MIDI files are a lot smaller than audio files like MP3s, WMAs, or WAVs. MIDI files are small and manageable enough that it's not uncommon to find them embedded in web pages, adding a sonic element to the surfing experience. MIDI files usually appear with the ".MID" filename extension.

8. MP2: MP2 stands for MPEG Audio Layer II or MPEG2 Audio, which used on VCDs, SVCDs and can be used DVDs. View MP2 to MP3 Converter.

9. MP3: MP3 stands for MPEG1 (or MPEG2) Audio Layer III. Too often people refer MP3 as MPEG3, which is incorrect, because such format doesn't even exist. MP3 is a popular compression format used for audio files on computers and portable devices. It is a method to store good quality audio into small files by using psychoacoustics in order to get rid of the data from the audio that most of the humans can't hear. MP3's bitrates vary from $8 \mathrm{kbps}$ to 320kbps. A typical MP3 file encoded at $128 \mathrm{kbps}$ is near CD quality. MP3 audio is increasingly being used in video production coupled with various MPEG4 video codecs like divx. View CD to MP3 Ripper,DVD to MP3 Ripper, and MP3 to WAV Converter.

10. M3U: M3U is a special type of metafile playlist that is used with MP3 files that have an .mp3 file extension. The .m3u files list one MP3 or other media file on each line, normally with full path or URL to the file. If the .m3u file is loaded to an MP3 player, the player normally plays the list of media files in the order they are listed in the playlist (unless options such as "randomize" have been selected in the MP3 player).

11. MP3 ID3 Tag: An MP3 ID3 Tag is information stored at the end of an MP3 file. The tag can contain information about the Title/Songname, Artist, Album, Year, Comment, and Genre in version 1 and also Track in version 1.1. A proposed Version 2 is out which would be extendable to include more information and pictures. View Audio Converter, convert M4A to MP3,MP4 to MP3, MP3 to WAV, etc, and supporting ID3 tag.

12. ID3: ID3 is a small piece of information stored physically inside the MP3 file (in the beginning or in the end of the file, depends on ID3's version). ID3 tags can contain various information about the MP3, like album name, song name, artist, original artist, genre, composer, releasing year, additional comment fields, etc.. Nowadays ID3s are de facto in audio world and they can be added to most of the audio formats and even to certain video formats in order to provide additional information of the file. View CD Ripper, rip audio CD to MP3. It supports ID3 ver 1 and ver 2 tag.

13. Ogg: Ogg is the umbrella for a group of several related multimedia and signal processing projects that are open source and royalty free. Development of these projects is controlled by Xiph.org.

14. Ogg Vorbis: Ogg Vorbis is an "open-source" digital audio compression format. like MP3, It is a "lossy" compression system, removing frequencies deemed inaudible. Both formats offer variable-bitrate encoding options, for better efficiency. But the algorithms Ogg Vorbis uses to decide which information to discard differ from those used by MP3. Proponents claim that the Ogg Vorbis format outperforms MP3, producing 
15. files that are significantly smaller than MP3s of similar sound quality (or files that sound better than similarly sized MP3s). View Ogg Converter.

16. VQF: VQF is one of the "alternative" audio compression formats back in 1990s that was aimed to take over MP3 by providing better audio quality than MP3 with lower bitrate. Failed miserabely due various reasons, most notably because of restrictive licensing. Nowadays the only serious alternatives to MP3 are probably Ogg Vorbis and Microsoft's WMA.

17. WAV: WAV is a standard audio format for Windows operating systems, often used for storing highquality, uncompressed sound. WAV files can contain CD-quality audio signals. However, CD-quality WAV files require relatively large amounts of memory. WAV files are probably the simplest of the common formats for storing audio samples. Unlike MPEG audio and other compressed formats, WAVs store samples "in the raw" where no pre-processing is required other that formatting of the data. The WAV file consists of three "chunks" of information: The RIFF chunk which identifies the file as a WAV file, The FORMAT chunk which identifies parameters such as sample rate and the DATA chunk which contains the actual data (samples). View MP3 WAV Converter.

18. WMA: WMA stands for Windows Media Audio. WMA, an audio format owned by Microsoft, is a part of Microsoft's Windows Media technology, which includes Microsoft's Digital Rights Management tools, Windows Media Video encoding technology and Windows Media Audio encoding technology. Windows Media Audio is one of today's most popular Net audio formats. Though not as popular as MP3, WMA tends to outperform MP3 in the area of sound quality, particularly with files encoded at lower bitrates like 64 or 96 Kbps. This performance advantage makes it handy for applications like portable digital audio players, where total play time is limited by a finite amount of internal memory.

\section{What is Streaming Music?}

\section{Overview of Audio Streaming}

Streaming Music, or more accurately known as streaming audio, is a way of delivering sound without the need to download files of different audio formats.

\section{What is streaming audio?}

Typically, if you want to listen to music, or any other type of audio, you need to download an audio file in a format such as an MP3, WMA, AAC, OGG, or Flac for example. However, using a streaming delivery method, there isn't the need to first download a complete file. The basic way that this process works is that the audio file is delivered in small 'packets' in order for the data to be buffered on your computer and played virtually straight away. As long as their is a steady stream of these packets delivered to your computer, you'll hear the sound without any interruptions. This method of listening to music is particularly useful for situations that need instantaneous delivery, such as live broadcasts or music services that typically provide 30 -second music clips before you buy.

\section{Windows Multipoint Server}

Windows MultiPoint Server 2011 is the second multi-server version of Windows. It is mostly used in educational institutions that allow multiple users to simultaneously share a single computer. Users have their own independent and familiar Windows computing experience, using their own monitor, keyboard and mouse directly connected to the host. The Windows MultiPoint Server 2011, so that more users to access technology at a lower total cost of ownership.

Designed for non-technical users, it is simple to manage and use. Unlike other similar solutions on the market, Windows MultiPoint Server 2011 based on the latest Windows technology and thus can run Windows applications.

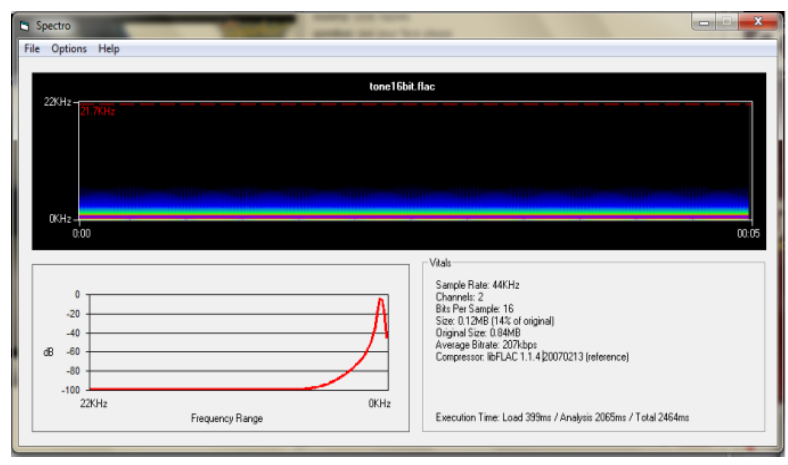




\section{Proposed Methodology}

In this proposed work we try to analyze three different kind of audio streams namely WMV, FLAC, MP3 for cloud audio streaming, in different environmental conditions. All the videos are streamed using cloud server Windows Multipoint Server 2011.

Scenario 1: In this scenario we try to access FLAC file on three different conditions

a. In this condition the FLAC file on wired network of the proposed cloud.

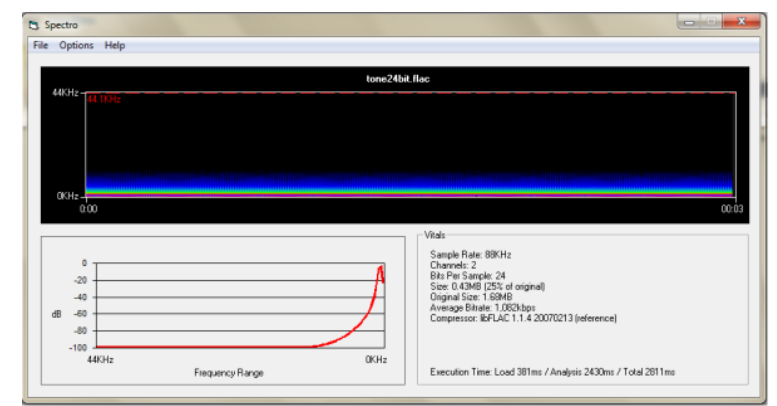

Fig 1 FLAC access on WIRED CLOUD NETWORK

b. In this condition the FLAC file on same server of the cloud.

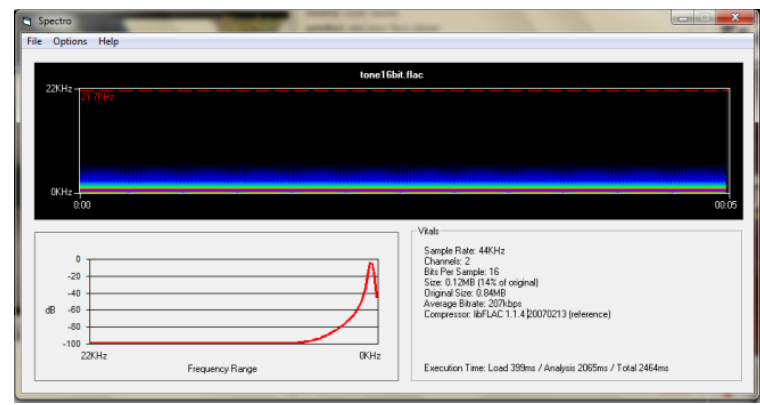

Fig 2 FLAC access on CLOUD SERVER

c. In this condition the FLAC file is accessed on wireless network of a cloud.

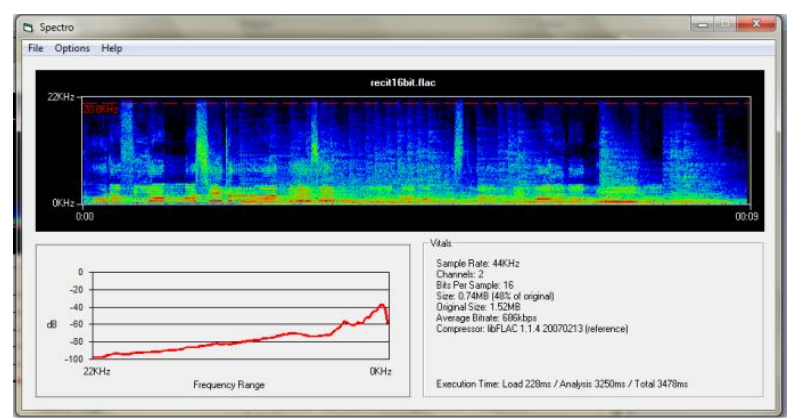

Fig 3 FLAC access on WIRELESS CLOUD NETWORK

Scenario 2: In this scenario we try to access WAV file on three different conditions a. In this condition the WAV file on wired network of the proposed cloud.

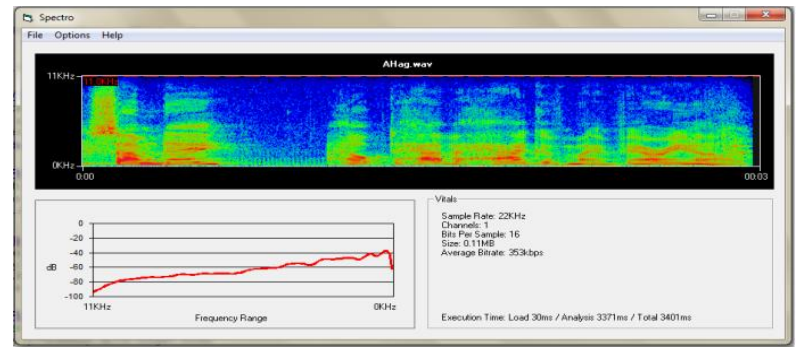

Fig 4 WAV access on WIRED CLOUD NETWORK 
b. In this condition the WAV file on wireless network of the proposed cloud.

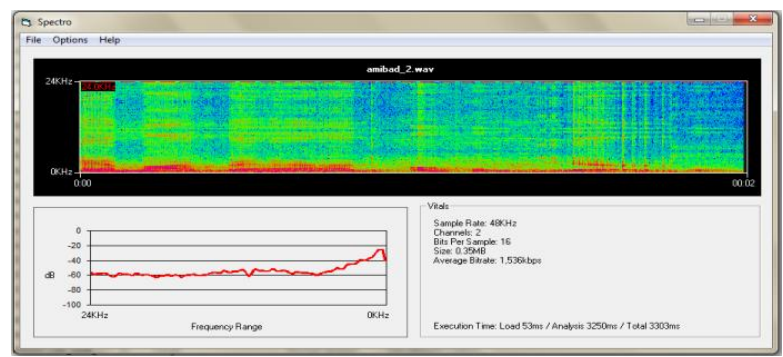

Fig 5 WAV access on WIRELESS CLOUD NETWORK

c. In this condition the WAV file on same server of the cloud.

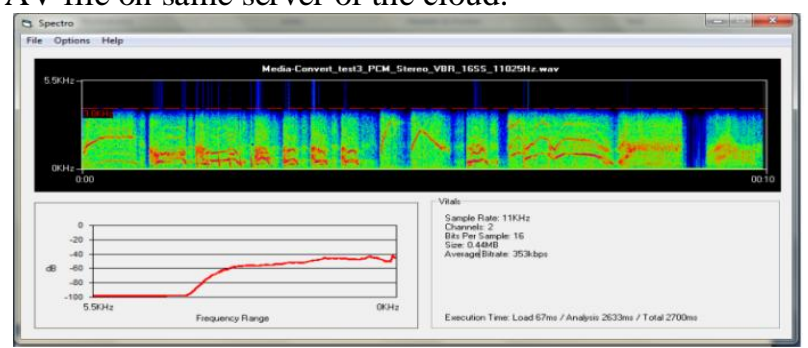

Fig 6 WAV access on CLOUD SERVER

Scenario 3: In this scenario we try to access MP3 file on three different conditions a. In this condition the MP3 file on wired network of the proposed cloud.

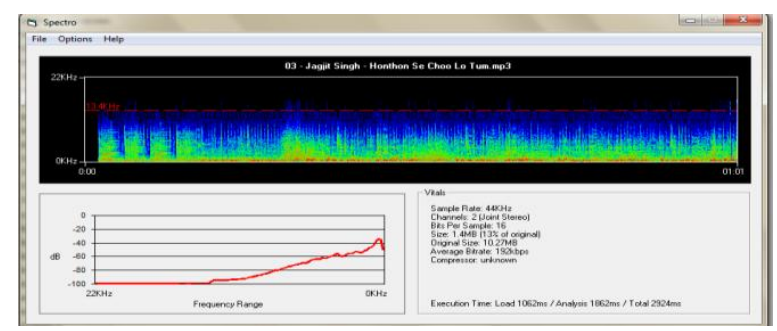

Fig 7 MP3 access on WIRED CLOUD NETWORK

b. In this condition the MP3 file on wireless network of the proposed cloud.

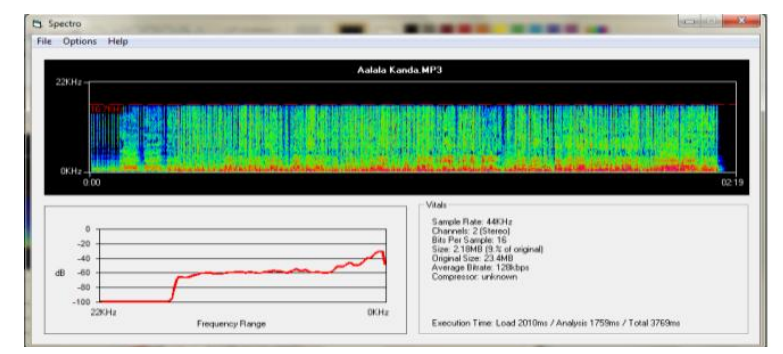

Fig 8 MP3 access on WIRELESS CLOUD NETWORK

c. In this condition the MP3 file on cloud server

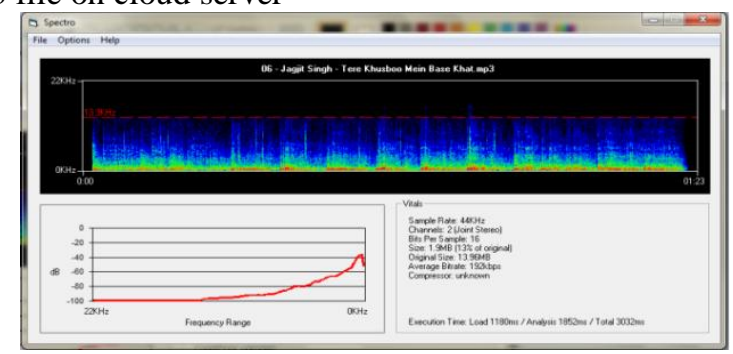

Fig 9 MP3 access on CLOUD SERVER 


\section{Conclusion \& Future Work}

As audio has been used as one of the best medium of communication today in growing IT scenario, various audio formats are in use for the same. and MP3.

In this work we are trying to analyze the performance of two of the audio Codecs namely WAV, FLAC

Whole work has been divided into 3 stages, namely stage 1 performance of FLAC, stage 2 performance of WAV and stage 3 performance of MP3.

Conclusions of all 3 stages' are as follows:

\section{Stage 1}

In this work we have tried to compare one of the codec format namely FLAC, audio in real time streaming scenario with various environment conditions.

Above analysis has been done for 3 different scenarios of single user in different environments.

From the above results following can be easily concluded:

1. The performance of FLAC is worst in wireless network

2. FLAC gives quite good bit rate at low frequency too.

3. The signals are lost least.

4. FLAC gives best performance in wired network distance access local machine access is not for cloud performance.

\section{Stage 2}

In this work we have tried to compare one of the codec format namely WAV, audio in real time streaming scenario with various environment conditions.

Above analysis has been done for 3 different scenarios of single user in different environments.

From the above results following can be easily concluded:

1. The performance of WAV is worst in wireless network

2. WAV gives very poor bit rate at low frequency too.

3. The signals are lost maximum.

4. The WAV gives worst performance of the all.

5. WAV gives best performance in wired network distance access local machine access is not for cloud Stage 3 performance.

In this work we have tried to compare one of the codec format namely MP3, audio in real time streaming scenario with various environment conditions.

Above analysis has been done for 3 different scenarios of single user in different environments.

From the above results following can be easily concluded:

1. The performance of MP3 is worst in wireless network

2. MP3 gives better bit rate at low frequency than WAV.

3. The signals are lost but more than FLAC.

4. MP3 gives best performance in wired network distance access local machine access is not for cloud performance.

\section{Concluding Summary}

By upper conclusions this can be easily interpreted that FLAC is best audio coded available as on date for streaming, whilst WAV is the worst.

\section{Future Work}

Cloud is the latest platform available in the sector to ensure availability of resources online in a closed or an open network.

Audio streaming has been used by many organizations to conduct various meeting in their cloud.

The quality of audio helps in easy understanding of the message communicated over the stream.

Though this work surround around some environments, In future analysis can be done for other audio formats also; results of two or more audio formats may be compared to find out the quality of delivery in a cloud.

Various environmental and quality metric parameters may also be added to prove the efficiency of audio streams. networks.

Current work is on streaming of normal audio, this work may also be carried out on secure audio

Even more improved codec and audio streaming mechanisms may be developed to get better quality of delivery in wireless networks 
[1] Dave Marshall, 1999

\section{References}

[2] Sue Smith, HRIS, Chron, 2013

[3] Fisher, B. and Schroeder, U. (1999). http://www7.tomshardware.com/audio

[4] Rice Library, "Audio Formats:A Guide to Understanding Audio Containers \& Codecs".

[5] Winxdvd resource centre, "What is MPEG", review article.

[6] Junaid Afzal et. al. ,"Audio Streaming over MBMS: A System Design Approach" JOURNAL OF MULTIMEDIA, VOL. 1, NO. 5, AUGUST 2006

[7] Fouquet M. et. al. "Cloud Computing for the Masses" Position paper. U-NET'09, December 1, 2009, Rome, Italy.

[8] Elecard Audio quest, 2008

[9] Nafaa A et. al. "A dependable multisource streaming system for peer-to-peer -based audio on demand services provisioning" Multimedia Tools Appl (2012) 59:169-220

[10] Chikkerur S. et. al., "Objective Audio Quality Assessment Methods: A Classification, Review, and Performance Comparison", IEEE TRANSACTIONS ON BROADCASTING, VOL. 57, NO. 2, JUNE 2011

[11] Hartsell, T., \& Yuen, S. (2006). Audio streaming in online learning. AACE Journal, 14(1), 31-43.

[12] HSU W.H. et. al. "QoS/QoE Mapping and Adjustment Model in the Cloud-based Multimedia Infrastructure" IEEE SYSTEMS JOURNAL.

[13] "On buffer requirements for store-and-forward audio on demand service circuits" IEEE. Retrieved 1991.

[14] Rahmalki, "Perfromance Analysus of multimedia streaming over internet", 2006

[15] Michael Armbrust, Armando Fox, Rean Griffith, Anthony D. Joseph, Randy H. Katz, Andrew Konwinski, Gunho Lee, David A. Patterson, Ariel Rabkin, Ion Stoica, and Matei Zaharia. Above the clouds: Aberkeley view of cloud computing. Technical report, EECS Department, University of California, Berkeley, Feb 2009.

[16] V. Bhaskaran and K. Konstantinides. Image and Audio Compression Standards: Algorithms and Architectures. Kluwer Academic Publishers, Boston, Massachusetts, 1997.

[17] Hamann C.J., Roitzsch M., Reuther L., Wolter J., and Hartig H. Probabilistic admission control to govern real-time systems under overload. In 19th Euromicro Conference Real-Time Systems, pages 211-222, 2007.

[18] Andrey Khorlin. Scheduling in distributed stream processing systems. Master's thesis, 2006.

[19] G. Morrison. Audio transcoders with low delay. In IEICE Trans. Communication, pages 963-969, 1997.

[20] Junaid Afzal, Thomas Stockhammer, Taigo Gasiba, Wen Xu Audio Streaming over MBMS: A System Design Approach, JOURNAL OF MULTIMEDIA, VOL. 1, NO. 5, AUGUST 2006

[21] A. Shokrollahi, "Raptor codes," Digital Fountain, Tech. Rep. DR2003-06-001, Jun. 2003.

[22] M. Luby, M. Watson, T. Gasiba, T. Stockhammer, and W. Xu, "Raptor codes for reliable download delivery in wireless broadcast systems," in Proc. of Consumer and Communications Networking Conference (CCNC), Las Vegas, NV, USA, Jan. 2006.

[23] Advanced Audio Coding for Generic Audiovisual Services, ITU-T and ISO/IEC JTC 1, 2003.

[24] S. Wenger, T. Stockhammer, M. Hannuksela, M. Westerlund, and D. Singer, "RTP payload format for H.264 audio," Internet Engineering Task Force (IETF)," RFC3984, Feb. 2005.

[25] 3GPP TS 26.346 V6.1.0, Technical Specification Group Services and System Aspects; Multimedia Broadcast/Multicast Service; Protocols and Codecs, June 2005.

[26] H. Jenkac, T. Stockhammer, and W. Xu, "Cross-Layer Issues and Forward Error Correction for Wireless Audio Broadcast," in Proc. of 16th Annual International Symposium on Personal Indoor and Mobile Radio Communications (PIMRC), Berlin, Germany, Sept. 2005. 